\title{
An Analysis of Die Swell with Rolie-Poly Model
}

\author{
Yu Cao ${ }^{1, a}$, Qian Wang ${ }^{1, b}$ Miao Wang ${ }^{1, c}$ Liyang $\mathrm{Xu}^{1, \mathrm{~d}}$ Xinhai $\mathrm{Xu}{ }^{1, \mathrm{e}, *}$ \\ ${ }^{1}$ State Key Laboratory of High Performance Computing, National University of Defense Technology, \\ Changsha, Hunan, China \\ aemail: caoyu@nudt.edu.cn, bemail: wangqian @nudt.edu.cn, bemail: mercury.miao @nudt.edu.cn \\ bemail: liyang_xu@163.com, bemail: xuxinhai@nudt.edu.cn
}

Keywords: Roli-Poly model; Polymer melts; Oldroyd-B model

\begin{abstract}
Previous efforts on die swell issues mainly focus on linear models such as Oldroyd-B, Linear PTT. However, the characteristic of Rouse Linear Entangled Polymers (Rolie-Poly) model, as a simple model derived from tube-based molecular theory, is rarely discussed before. Compared with other constitutive models, the Rolie-Poly model did a better job in predicting the dynamics of entangled polymer melts problems. In this paper, we analyzed the role of parameters in Rolie-Poly model when determining the behavior of die swell in 2D cases. Results show that the velocity in the die and the shape of the free surface are both totally different from Oldroyd-B model. It seems that the reptation relaxation time plays a dominant role in determining the final result of the extrusion. Moreover, the input velocity might have different influences under different conditions.
\end{abstract}

\section{Introduction}

In recent years, a thorough and comprehensive understanding of the behavior of viscoelastic flows is in an urgent need in both academic and industrial fields, and a large variety of constitutive models have been developed to reproduce experimental results. Actually speaking, the rheological response of viscoelastic fluids is so complex that no existed model could fully explain their motion precisely yet. Current non-linear viscous and elastic effects mainly include memory effects, normal stress differences, strain rate dependent viscosity, stress relaxation. Furthermore, the mechanism of stress relaxation include reptation, chain-stretch, self-consistent constraint release (CR), contour length fluctuations (CLF), convective constraint release (CCR), etc. A good model need to describe the interplay of all these effects in a proper manner.

The Rolie-Poly model [1], derived from tube-based molecular theory [2], is a simple equation which is comprised of two relaxation times: stretch relaxation and orientation relaxation. It was widely accepted in entangled linear polymer system simulation which incorporated reptation of the polymers within the tubes, the stretching of the polymer chains and CCR effects. Unlike for the Oldroyd-B model, the second normal stress difference is non-zero. Furthermore, numerical extrusion simulations with Oldroyd-B model have to tackle with high Weissenberg number problem [3]. Tanner [4, 5] is famous for the prediction of the swelling ratio in theory. His formula was derived by modeling the elastic effect as a K-BKZ integral equation, and according to his formula, the swelling ratio is a function of the recoverable shear. However, Rolie-Poly model has two relaxation times and a non-zero second normal stress difference, which means his formula is no more suitable for swelling prediction.

Regarding the basic methodology to simulate free surface issues like die swell, best known approaches include interface capturing methods and interface tracking methods. Interface capturing methods , like Volume of fluid (VOF) method [6], Level set method [7], Marker and Cell (MAC) method [8], rebuild the interface by numerical schemes. While interface tracking method could track the interface directly and precisely by computational grids. Generally speaking, the interface capturing method could overcome distortion cases and the interface tracking method generally owns a higher accuracy with the same number of computational cells. Arbitrary Lagrangian-Eulerian (ALE) method [9] could not track the exact interface directly by grids, but also could only avoid mesh distortion by constraining the moving direction of the grids. However, ALE method is usually 
combined with finite element method (FEM) [10, 11]. Tukovic [12] firstly developed an method to combine ALE with finite volume method (FVM) in OpenFOAM (Open Source Field Operation and Manipulation) [13]. The limitation of his method is that it could only tackle with Newtonian fluid problems. Moreover, additional meshes were introduced which increased the computational cost and complexity.

In this paper, extrusion simulations were implemented with the interface tracking method we proposed before [14] in widely used CFD simulation software OpenFOAM[15-17]. We mainly discussed about the performance of die swell with Rolie-Poly model with different parameters in its constitutive equation. Significant distinctions were seen as described in the following sections.

\section{Methodology}

Mathematical model Considering incompressible, isothermal and inertia-less flows, the governing equations for the Rolie-Poly model, including the momentum equation, mass conservation, and constitutive equation in ALE form, are given by:

$$
\begin{gathered}
-\nabla p+\nabla \cdot \tau+\eta_{s} \Delta u=0 \\
\nabla \cdot u=0 \\
\frac{\partial \boldsymbol{C}}{\partial t}+(u-w) \cdot \nabla \boldsymbol{C}=(\nabla u)^{T} \cdot \boldsymbol{C}+\boldsymbol{C} \cdot \nabla u-\frac{1}{\tau_{d}}(\boldsymbol{C}-\boldsymbol{I})-\frac{2(1-\sqrt{2 / \operatorname{tr} \boldsymbol{C}})}{\tau_{R}}\left(\boldsymbol{C}+\beta\left(\frac{\operatorname{tr} \boldsymbol{C}}{2}\right)^{\delta}\right)(\boldsymbol{C}-\boldsymbol{I})
\end{gathered}
$$

Where $p$ is the pressure, $\tau$ is the polymeric contribution for stress tensor, $\eta_{s}$ is the solvent viscosity. The constitutive equation of Rolie-Poly model is a little bit different from the original on proposed by Likhtman [1], because our simulations is based on 2D cases which means the dimensionality of free moving for a particle is two [18]. In the 2D Rolie-Poly equation, $u$ is the fluid velocity, and $w$ is the velocity of the boundary surface. $\tau_{d}$ and $\tau_{R}$ represents the reptation time and Rouse time respectively. $\beta$ and $\delta$ here are used to describe the behaviour of CCR. $\beta$ often varies from 0 to 1 , while $\delta$ often takes the value of -0.5 according to experimental results. $\boldsymbol{I}$ is the unit tensor. $\boldsymbol{C}$ is the polymer conformation tensor which determines the polymeric component of the stress $\tau$. The relationship between them is given by

$$
\tau=\frac{\eta_{p}}{\tau_{d}}(\boldsymbol{C}-\boldsymbol{I})
$$

where $\eta_{p}$ is the polymer viscosity. In order to avoid the unexpected numerical instability caused by subtracting a constant during intermediate procedures, we introduced a new parameter, the polymeric strain $\boldsymbol{W}=\boldsymbol{C}-\boldsymbol{I}$, to take the place of the polymer conformation tensor. By substituting the new parameter into Eq. 3, we could obtain:

$$
\frac{\partial \boldsymbol{W}}{\partial t}+(u-w) \cdot \nabla \boldsymbol{W}=2 D+(\nabla u)^{T} \cdot \boldsymbol{W}+\boldsymbol{W} \cdot \nabla u-\frac{1}{\tau_{d}} W-\frac{2(1-\sqrt{2 / \operatorname{tr} \boldsymbol{W}})}{\tau_{R}}\left(\boldsymbol{I}+\boldsymbol{W}+\beta\left(\frac{\operatorname{tr} \boldsymbol{W}}{2}\right)^{\delta} \mathbf{W}\right)
$$

where $\boldsymbol{D}$ is the rate of deformation tensor, which can be expressed as $\boldsymbol{D}=\left(\nabla u+(\nabla u)^{T}\right) / 2$. When $\tau_{R} \rightarrow \infty$, the Rolie-Poly model would reduce to Oldrod-B model.

Geometry and initial conditions This work took the typical half geometry for die swell simulation like [19]. Details about the geometric shape and the boundary conditions is illustrated in Fig. 1 . We choose the radius of the die $\mathrm{L}=1$ as the characteristic length, the length of the upstream and the downstream are chosen as $8 \mathrm{~L}$. We assumed that the flow would fully developed and have reached a steady state at both the exit of the die and the end of the flow. At the inflow, we impose 
the velocity profile along $\mathrm{x}$ direction as: $u_{i n}=u_{\max }\left(1-\mathrm{y}^{2}\right)$. Thus the average velocity of the inflow is $\bar{u}=(2 / 3) u_{\max }$. In order to make a close comparison with former literatures [19-21], we choose $u_{\max }=1.5$ if not specified. The boundary condition at the centerline is set as symmetry because the geometry is rotationally symmetric. Velocity boundary conditions are no-slip at the wall and zero derivative at the remaining boundaries. As for the pressure fields, the condition are assumed to be zero at the outlet and zero gradient at the wall and inlet. Boundary condition at the free surface is a bit more complex as it should change dynamically during the simulation so that the force balance could always satisfied during the extrusion. Here $\sigma=-p I+2 \eta_{s} D+\tau$ is the total stress tensor. Though there is no analytical solution for a fully developed Poiseuille flow for the Rolie-Poly model, we supposed that the polymeric stress is already fully relaxed at inlet, i.e. $\tau_{\text {in }}=0$.

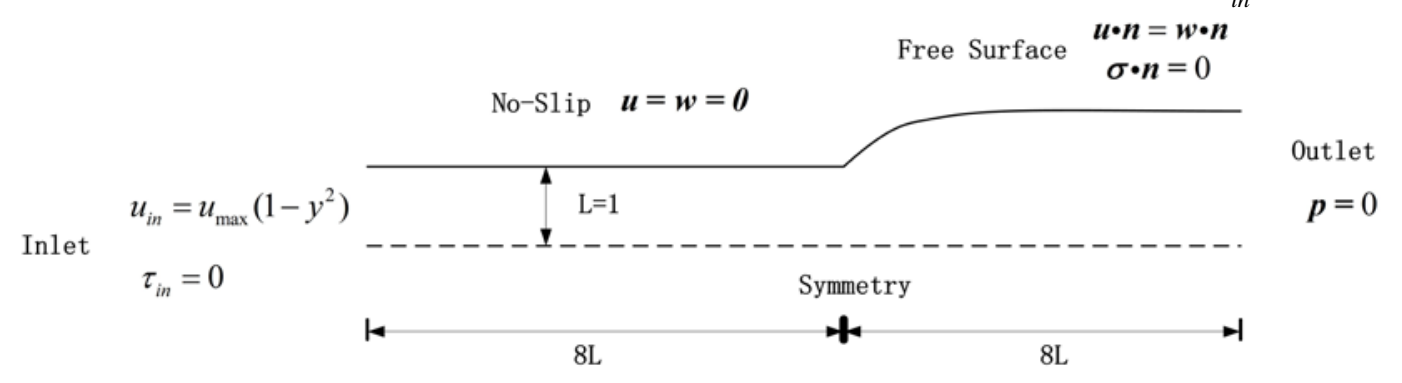

Fig. 1. Die swell flow configurations

Numerical algorithm In order to enhance the numerical stabilization of the momentum equation, a typical numerical methodology called discrete elastic split stress (DEVSS) [22, 23] was adopted. Essentially, it added an artificial extra force in the equation which would solved implicitly, meanwhile an additional source term expressed explicitly with the same value would be subtracted. The algorithm may be illustrated as the following:

$$
\left(\eta_{s}+\eta_{a}\right) \Delta u+\left(\nabla \cdot \tau-\eta_{a} \Delta u\right)=-\nabla p
$$

where $\eta_{a}$ is the added artificial viscosity and is usually chosen as $\eta_{p}$. Substituting the polymeric strain $W$ into Eq. 6 and dividing a constant $\tau_{d} / \eta_{p}$ for each elements in the equation, the final momentum equation is given by:

$$
\frac{\tau_{d}}{\eta_{p}}\left(\eta_{s}+\eta_{a}\right) \Delta u+\nabla \cdot \boldsymbol{W}-\left(\frac{\eta_{a} \tau_{d}}{\eta_{p}}\right) \Delta u=-\frac{\tau_{d}}{\eta_{p}} \nabla p
$$

\section{Results and discussion}

In this section, the influence if each parameter in Rolie-Poly model, including $\tau_{d}, \tau_{R}$ and $\beta$, is analyzed carefully. In addition, the behavior of different fluids under various velocity is studied. Unless specifically stated, The parameters used in the simulations are $\tau_{R}=1, \tau_{d}=10, \beta=0$, $\delta=-0.5, \eta_{s}=0.1, \eta_{p}=0.9$.

Influence of $\boldsymbol{\tau}_{\boldsymbol{d}}$ We firstly investigated the influence of $\tau_{d}$ by fixing $\tau_{R}=1$ and choosing $\tau_{d}$ as $10,100,1000$. Velocity profiles in the middle of the die (at $\mathrm{x}=-4$ ) are shown in Fig. 2. When $\tau_{d}$ is large (e.g. $\tau_{d} \geq 100$ ), the constitutive equation is similar the Oldroyd-B model, thus, the velocity profile of Rolie-Poly model is close to the one of Oldroyd-B model. However, when $\tau_{d}$ is relatively small $\left(\tau_{d}=10\right)$, the shear rate near the wall $\gamma_{w}$ would become larger. The shape of the out flow are also quite different from Oldroyd-B model. Generally, the free surface would swell 
gradually and reach a plateau at last, however, for the case of $\tau_{d}=10$, it would decline gradually after a peak of the surface. For the case of $\tau_{d}=100$, the surface is kept swelling which means the extrudate part is probably insufficient long. In terms of the swelling ratio, it would increase at first, and decrease afterwards.

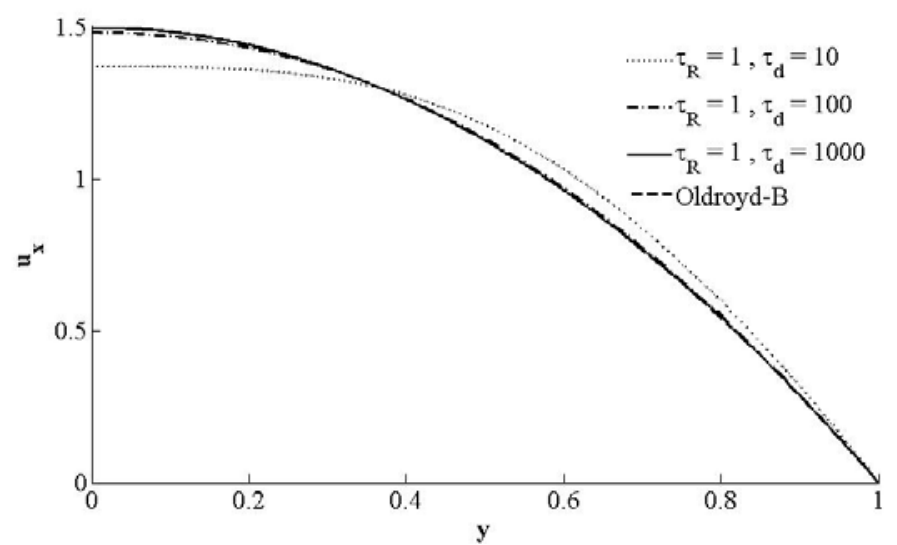

Fig. 2. Velocity profile in the middle of the die

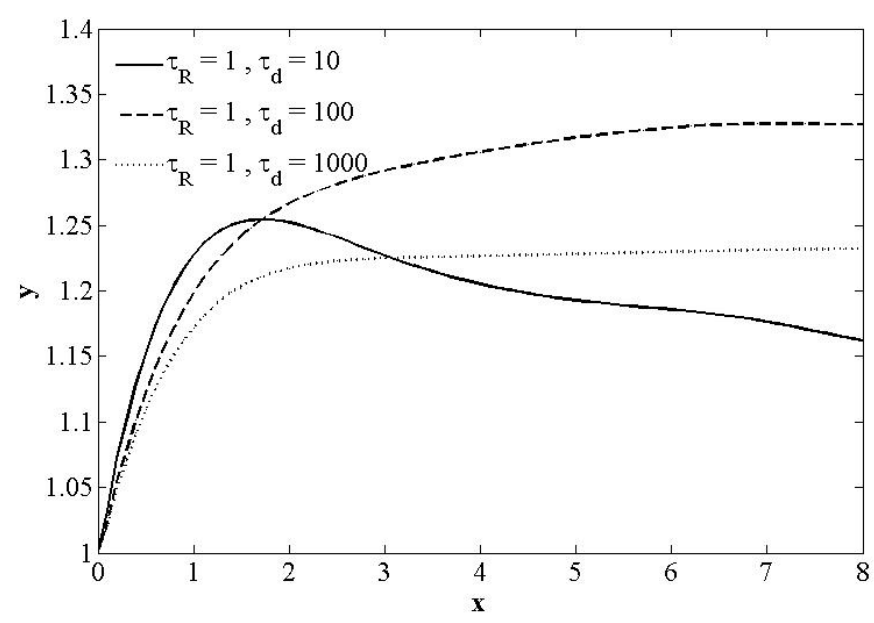

Fig. 3. Free surface shape with different $\tau_{d}$

Influence of $\boldsymbol{\tau}_{\boldsymbol{R}}$ Table 1 shows the influence of $\tau_{R}$ by maintaining $\tau_{d}=10$. When $\tau_{R}$ is very small, the parameter $\tau_{R}$ has no significant influence on the swelling ratio. While when $\tau_{R}$ is growing larger, it would result in an increase of the swelling ratio gradually. Relatively speaking, it seems that $\tau_{R}$ has a little influence than $\tau_{d}$ in extrusion cases.

Table 1. The swelling ratio with different $\tau_{R}$

\begin{tabular}{|l|l|}
\hline$\tau_{R}$ & $\mathrm{~S}_{\mathrm{R}}$ \\
\hline 0.01 & 1.201 \\
\hline 0.1 & 1.200 \\
\hline 0.5 & 1.221 \\
\hline 1 & 1.261 \\
\hline
\end{tabular}

Influence of $\boldsymbol{\beta}$ From Table 2, It seems that $\beta$ also has little influence on the swelling ratio. The variation of the swelling ratio between $\beta=0$ and $\beta=1$ is no more than $1.3 \%$ and $5 \%$ when $\tau_{d}=10, u=4$ and $\tau_{d}=100, u=1$ respectively. Moreover, from the case of $\tau_{d}=10$ and $u=4$, whether a larger $\beta$ would result in a smaller swelling ratio is unsure yet. 
Table 2. The swelling ratio under different parameters

\begin{tabular}{|l|l|l|l|}
\hline$\left(\tau_{d}, \beta, u\right)$ & $\mathrm{S}_{\mathrm{R}}$ & $\left(\tau_{d}, \beta, u\right)$ & $\mathrm{S}_{\mathrm{R}}$ \\
\hline$(10,0,4)$ & 1.659 & $(100,0,1)$ & 1.328 \\
\hline$(10,0.5,4)$ & 1.669 & $(100,0.5,1)$ & 1.290 \\
\hline$(10,1,4)$ & 1.641 & $(100,1,1)$ & 1.267 \\
\hline
\end{tabular}

Influence of average velocity Generally, a high average velocity means a high Weissenberg number, which would result in a large swelling ratio. But it is not always the case when $\tau_{R}$ and $\tau_{d}$ are very small. Table 3 shows that when $\tau_{R}=1$ and $\tau_{d}=10$, the swelling ratio would increase with the augment of average velocity. However, when $\tau_{R}=0.001$ and $\tau_{d}=0.01$, the trend of the variation is totally opposite. This is because the elastic effect is quite small when $\tau_{R}$ and $\tau_{d}$ are very small, which means the property of current fluid is quite similar to the one of Newtonian fluid.

Table 3. The swelling ratio under different average velocity with various $\left(\tau_{R}, \tau_{d}\right)$

\begin{tabular}{|l|l|l|l|l|l|}
\hline$\left(\tau_{R}, \tau_{d}\right)$ & Average velocity & $\mathrm{S}_{\mathrm{R}}$ & $\left(\tau_{R}, \tau_{d}\right)$ & Average velocity & $\mathrm{S}_{\mathrm{R}}$ \\
\hline \multirow{3}{*}{$(1,10)$} & 1 & 1.254 & \multirow{3}{*}{$(0.001,0.01)$} & 2 & 1 \\
\cline { 2 - 5 } & 2 & 1.408 & 2 & 1.171 \\
\cline { 2 - 3 } & 4 & 1.659 & & 4 & 1.167 \\
\hline
\end{tabular}

\section{Conclusion}

This paper analyzed the influence of each parameter in Rolie-Poly model. The results showed that the velocity in the die and shape of the extrudate part is totally different from Oldroyd-B model. Besides, It seemed that the reptation relaxation time $\tau_{d}$ dominates the extrusion behavior. Moreover, increasing the inlet velocity might decrease the swelling ratio when the two relaxation time are small.

\section{Acknowledgement}

We are grateful to the fund from the National Natural Science Foundation of China (No.61221491, No.61303071, and No.61303068), and Open fund from State Key Laboratory of High Performance Computing (No.201303-01, No.201303-02).

\section{References}

[1] Likhtman, A.E. and R.S. Graham, Simple constitutive equation for linear polymer melts derived from molecular theory: Rolie-Poly equation. Journal of Non-Newtonian Fluid Mechanics, 2003. 114(1): p. 1-12.

[2] Graham, R.S., A.E. Likhtman, and T.C.B. McLeish, Microscopic theory of linear, entangled polymer chains under rapid deformation including chain stretch and convective constraint release. Journal of Rheology, 2003. 47(5): p. 1171-1200.

[3] Tenchev, R., et al., Three dimensional finite element analysis of the flow of polymer melts. Journal of Non-Newtonian Fluid Mechanics, 2011. 166(5-6): p. 307-320.

[4] Tanner, R.I., Engineering Rheology. 2002, Oxford: Oxford University Press.

[5] Tanner, R.I., A theory of die-swell revisited. Journal of Non-Newtonian Fluid Mechanics, 2005. 129(2): p. 85-87.

[6] Favero, J.L., et al., Viscoelastic fluid analysis in internal and in free surface flows using the software OpenFOAM. Computers \& Chemical Engineering, 2010. 34(12): p. 1984-1993. 
[7] Osher, S. and J.A. Sethian, Fronts Propagating with Curvature-Dependent Speed - Algorithms Based on Hamilton-Jacobi Formulations. Journal of Computational Physics, 1988. 79(1): p. 12-49.

[8] Mckee, S., et al., The MAC method. Computers \& Fluids, 2008. 37(8): p. 907-930.

[9] Hirt, C.W., A.A. Amsden, and J.L. Cook, An arbitrary Lagrangian-Eulerian computing method for all flow speeds (Reprinted from the Journal of Computational Physics, vol 14, pg 227-253, 1974). Journal of Computational Physics, 1997. 135(2): p. 203-216.

[10]Fortin, M. and D. Esselaoui, A Finite-Element Procedure for Viscoelastic Flows. International Journal for Numerical Methods in Fluids, 1987. 7(10): p. 1035-1052.

[11]Rasmussen, H.K., Time-dependent finite-element method for the simulation of three-dimensional viscoelastic flow with integral models. Journal of Non-Newtonian Fluid Mechanics, 1999. 84(2-3): p. 217-232.

[12] Tukovic, Z. and H. Jasak, A moving mesh finite volume interface tracking method for surface tension dominated interfacial fluid flow. Computers \& Fluids, 2012. 55: p. 70-84.

[13] Jasak, H., OpenFOAM: Open source CFD in research and industry. International Journal of Naval Architecture and Ocean Engineering, 2009. 1(2): p. 89-94.

[14] Yu, C., et al., A new method to simulate free surface flows for viscoelastic fluid. Advances in Materials Science and Engineering, 2015. Submitted

[15] Xu, X.-H., et al., Multi-scale simulation of non-equilibrium phase transitions under shear flow in dilute polymer solutions. RSC Advances, 2015. 5(67): p. 54649-54657.

[16]Guo, X.-W., et al., Interface instabilities and chaotic rheological responses in binary polymer mixtures under shear flow. RSC Advances, 2014. 4(105): p. 61167-61177.

[17] Guo, X.-W., et al., Non-equilibrium steady states of entangled polymer mixtures under shear flow. Advances in Mechanical Engineering, 2015. 7(6): p. 1-10.

[18]Chung, C., et al., Numerical study of chain conformation on shear banding using diffusive Rolie-Poly model. Rheologica Acta, 2011. 50(9-10): p. 753-766.

[19] Crochet, M.J. and R. Keunings, Finite-Element Analysis of Die Swell of a Highly Elastic Fluid. Journal of Non-Newtonian Fluid Mechanics, 1982. 10(3-4): p. 339-356.

[20]Tome, M.F., et al., A finite difference technique for simulating unsteady viscoelastic free surface flows. Journal of Non-Newtonian Fluid Mechanics, 2002. 106(2-3): p. 61-106.

[21]Russo, G. and T.N. Phillips, Spectral element predictions of die-swell for Oldroyd-B fluids. Computers \& Fluids, 2011. 43(1): p. 107-118.

[22] Guenette, R. and M. Fortin, A New Mixed Finite-Element Method for Computing Viscoelastic Flows. Journal of Non-Newtonian Fluid Mechanics, 1995. 60(1): p. 27-52.

[23] Matallah, H., P. Townsend, and M.F. Webster, Recovery and stress-splitting schemes for viscoelastic flows. Journal of Non-Newtonian Fluid Mechanics, 1998. 75(2-3): p. 139-166. 